Fig. 2 - Simulation Results: Alternative Definitions

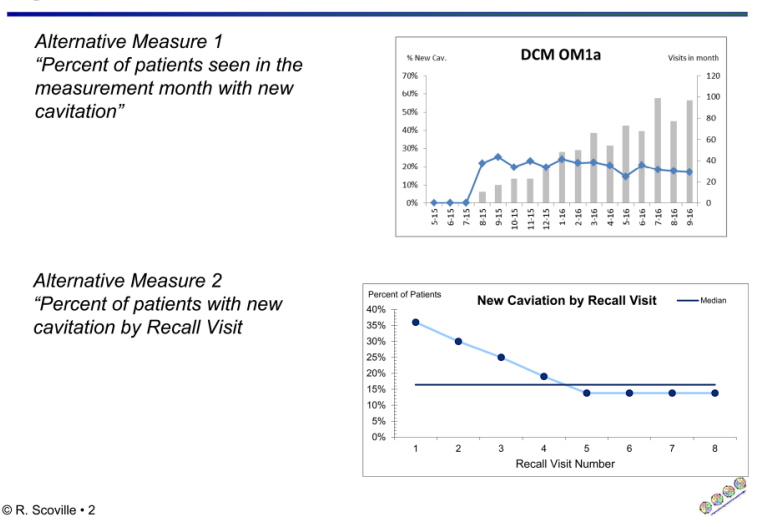

Figure 2 Simulation results: alternative definitions.

Fig. 3 - Results: Individual Team Data

\section{PREDICTING OUTCOME MEASURE PERFORMANCE THROUGH MONTE CARLO SIMULATION}

Richard Scoville, ${ }^{1}$ Manwai Ng, ${ }^{2}$ Gareth Parry, ${ }^{1}$ Abiola Faniyan, ${ }^{2}$ Adam Richman, ${ }^{3}$ Arthur Evans ${ }^{3} .{ }^{1}$ Institute for Healthcare Improvement, United States; ${ }^{2}$ Boston Children's Hospital, United States; ${ }^{3}$ Dentaquest Institute, United States

\subsection{6/bmjgs-2015-IHlabstracts. 19}

Background Improvement initiatives rely on measures to assess their impact. When outcomes are expressed as percentages, unanticipated changes to the denominator due to complexities and interactions among processes, population and contextual factors can result in measures that fail to register real improvements. For example a pediatric oral health collaborative focused on the incidence of new dental caries as a key measure. Despite uptake of process changes, a prevalence measure failed to register associated improvement in appointment rates (fig 1). Data exploration suggested the measure was confounded with exposure to process changes and recruitment of new patients.

Objectives To assess whether applying Monte-Carlo simulation can help project designers test the performance of outcome measures under an array of anticipated conditions.

Methods Data were obtained via a database application installed at each practice which calculated monthly collaborative

\section{Fig. 1- Original Measure}

"Percent of high-risk patients with
new caries at their most recent
visit."
Predicted
Observed
Based on
Simulated Data

